\title{
Sleep apnea and aortic dissection
}

\author{
PATRICK J HANLY MD FRCPC, DAVID FORD MSC \\ Sleep Laboratory, Department of Medicine, The Wellesley Hospital, Toronto, Ontario
}

\section{PJ HANLY, D FORD. Sleep apnea and aortic dissection. Can Respir J 1995;2(1):69-73.}

A 62-year-old man presented with obstructive sleep apnea, congestive heart failure and Cheyne-Stokes respiration. Further evaluation revealed a chronic dissecting aneurysm of the aorta causing vena caval obstruction. Surgical correction of the aneurysm dramatically improved ventricular function with resolution of Cheyne-Stokes respiration. Nasal continuous positive airway pressure corrected the obstructive sleep apnea and associated clinical features. The cardiopulmonary interactions between sleep apnea and aorlic dissection are discussed.

Key Words: Amrtic aneurysm, Cheyne-Stokes respiration, Sleep apnea

\section{Apnée du sommeil et dissection aortique}

RÉSUMÉ : Un homme de 62 ans présentait une apnée du sommeil obstructive, une insuffisance cardiaque et une respiration de Cheyne-Stokes. Un examen plus approfondi a révélé un anévrisme chronique disséquant de l'aorte causant une obstruction de la veine cave. Une chirurgie corrective de l'anévrisme a considérablement amélioré la fonction ventriculaire et a résolu la respiration de Cheyne-Stokes. La pression positive continue administrée par voie nasale a corrigé l'apnée du sommeil obstructive ainsi que les symptômes qui y étaient associés. Les interactions cardiopulmonaires de l'apnée du sommeil et de la dissection aortique sont examinées.
$\mathrm{C}$ HEYNE-STOKES RESPIRATION (CSR) IS A FORM OF CENtral sleep apnea caused by periodic changes in respiratory drive (1). It is characteristically seen in patients with severe congestive heart failure (2) and has not been reported in association with aortic aneurysm. Obstructive sleep apnea (OSA) is caused by upper airway instability during sleep and may contribute to left ventricular dysfunction in patients with co-existing heart disease (3). A case of a patient who had CSR due to vena caval obstruction associated with a dissecting aneurysm of the ascending aorta is presented. The patient also had OSA, which may have contributed to the development of his aortic dissection.

\section{CASE PRESENTATION}

History: A 62-year-old male presented with a long history of OSA and a recent history of congestive heart failure. He reported very loud, habitual snoring and excessive daytime sleepiness for 40 years. Initially, he had passive sleepiness but it became so severe that he had frequent road traffic accidents and discontinued driving. His wife had witnessed apneas during sleep for 20 years. The patient had been obese all his life and, on presentation, weighed $153 \mathrm{~kg}$ with a body mass index of $46 \mathrm{~kg} / \mathrm{m}^{2}$. He reported drinking 6 to $8 \mathrm{oz}$ of alcohol per day for several years.

The patient had been treated for hypertension lor 10 years. 
TABLE 1

Overnight polysomnography at presentation and 13 months following surgery, on and off nasal CPAP

\begin{tabular}{lccc}
\hline & $\begin{array}{c}\text { Before } \\
\text { surgery }\end{array}$ & $\begin{array}{c}\text { After surgery, } \\
\text { off CPAP }\end{array}$ & $\begin{array}{c}\text { After surgery, } \\
\text { on CPAP }\end{array}$ \\
\hline TST (h) & 4.87 & 2.3 & 3.6 \\
Sleep efficiency (\%) & 81 & 76 & 94 \\
Stage $1,2(\% T S T)$ & 98 & 86 & 69 \\
SWS (\%TST) & 0 & 14 & 4 \\
REM (\% TST) & 2 & 0 & 27 \\
AHI (/h) & 61 & 43 & 3 \\
PLM (/h) & 62 & 20 & 17 \\
AR (/h) & 53 & 26 & 14 \\
$\mathrm{SaO}_{2}$ (TST) & 82 & 85 & 92 \\
tCO $_{2}$ (TST) & 34 & 43 & 44 \\
\hline
\end{tabular}

AHl Apnea-hypopnea index; AR Arousal index; CPAP Continuous positive airway pressure; PLM Periodic leg movement index; REM Rapid eye movement sleep; $\mathrm{SaO}_{2}$ (TST) Mean oxygen saturation during TST; Stage 1.2 Stage 1 and 2 non-rapid eye movement sleep; SWS Slow wave sleep; $t C O_{2}$ (TST) Mean transcutaneous $P \mathrm{CO}_{2}$ in $m m H g$ during TST; TST Total sleep time

Seven years previously, he developed viral pericarditis and a small pericardial effusion, which was detected by echocardiography. This was complicated by atrial fibrillation, which was successfully cardioverted. Over the past few months, he developed increasing shortness of breath and peripheral edema. His medications included furosemide, digoxin, amiloride, enalapril and allopurinol.

Physical examination: The patient was a morbidly obese male. He had atrial fibrillation with a ventricular response of 150 beats/min. Blood pressure was $140 / 80 \mathrm{mmHg}$. Neck circumference was $51 \mathrm{~cm}$ and he had a large uvula and crowded oropharynx. Cardiovascular examination revealed an elevated jugular venous pressure, displaced left ventricular apex beat and severe, bilateral leg edema.

Overnight polysomnography: Electroencephalogram (EEG), electro-oculogram (EOG) and submental electromyogram (EMG), airflow, movement of the chest and abdomen (Respitrace), arterial oxygen saturation $\left(\mathrm{SaO}_{2}\right.$, by Ohmeda Biox 3740 pulse oximeter), transcutaneous $\mathrm{PCO}_{2}$ and electrocardiogram (ECG) were all monitored. Movement of the left and right leg were recorded independently from the anterior tibialis EMG via surface electrodes. All variables were continuously recorded on a polygraph (Model 78E, Grass Instruments, Massachusetts) at a paper speed of 10 $\mathrm{mm} / \mathrm{s}$. Transcutaneous partial pressure of carbon dioxide $\left(\mathrm{PCO}_{2}\right)$ was displayed on a slow recorder (paper speed 20 $\mathrm{cm} / \mathrm{h}$ ) synchronized to the Grass polygraph.

Polysomnograms were scored manually and sleep stage, and arousals were determined by established criteria using the EEG, EOG and EMG (4). Periodic leg movements were scored if they occurred independently of an arousal from sleep and were part of a series of four or more consecutive movements lasting 0.5 to $5.0 \mathrm{~s}$ with an intermovement interval of 5 to $90 \mathrm{~s}(5)$. CSR was defined as periodic breathing with central apnea or hypopnea alternating with hyperpnea in a crescendo/descrescendo pattern, and the duration of CSR

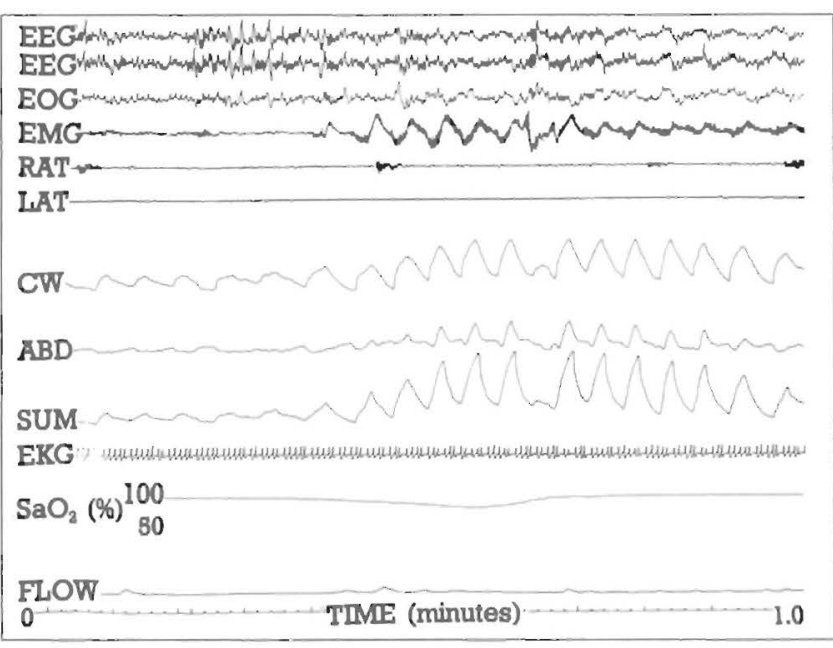

Figure 1) One minute of nonrapid eye movement steep demonstrating paradoxical breathing followed by hyperpnea with a crescendoldecrescendo pattern. EEG Electroencephalogram; EOG Electro-oculogram; EMG Submental electromyogram; RAT and LAT Right and left anterior tibialis EMG; CW Chest wall movement; $A B D$ Abdominal wall movement; SUM Sum of CW and ABD. EKG Electrocardiogram; $\mathrm{SaO}_{2}$ Oxygen saturation; FLOW Airflow at nose and moutl?

was expressed as a percentage of the total sleep time (TST). Mean oxygen saturation during sleep was calculated by averaging the high and low $\mathrm{SaO}_{2}$ for each $30 \mathrm{~s}$ epoch. Mean transcutaneous $\mathrm{PCO}_{2}\left(\mathrm{tCO}_{2}\right)$ during sleep was calculated from the average $\mathrm{tCO}_{2}$ over $36 \mathrm{~s}$ intervals.

The overnight sleep study revealed severe sleep apnea, periodic leg movements during sleep and rapid atrial fíbrillation (Table I). During nonrapid eye movement (NREM) sleep, there were features both of OSA, manifested by rib cage paradox, and CSR with a classic crescendo/decrescendo appearance (Figure I). During rapid eye movement (REM) sleep, apneas were clearly obstructive without evidence of CSR (Figure 2). Apneas were 30 to $40 \mathrm{~s}$ long during NREM sleep and up to $65 \mathrm{~s}$ long during the brief period of REM sleep. They were associated with severe hypoxemia and sleep disruption. Transcutaneous $\mathrm{PCO}_{2}$ was low during both wakefulness and sleep. The patient was immediately placed on nasal continuous positive airway pressure (CPAP) and a repeat sleep study demonstrated that $10 \mathrm{cmH}_{2} \mathrm{O}$ nasal CPAP corrected upper airway obstruction and maintained satisfactory oxygen saturation during sleep. Furthermore, once the upper airway was stabilized by nasal CPAP, CSR became more apparent with a typical crescendo/decrescendo appearance (Figure 3). This occurred only during NREM sleep and was associated with mild oxygen desaturation to between 85 and $90 \%$. The patient continued to have frequent periodic leg movements during sleep and rapid atrial fibrillation.

Pulmonary function: Spirometry showed a vital capacity of $3.2 \mathrm{~L}$ ( $78 \%$ predicted), and a forced expiratory volume in $1 \mathrm{~s}$ (FEV $)$ of $2.54 \mathrm{~L}$ ( $88 \%$ predicted), with a normal inspiratoryexpiratory flow-volume loop. Lung volumes (body plethysmography) were: total lung capacity $5.74 \mathrm{~L}$ ( $87 \%$ predicted), 


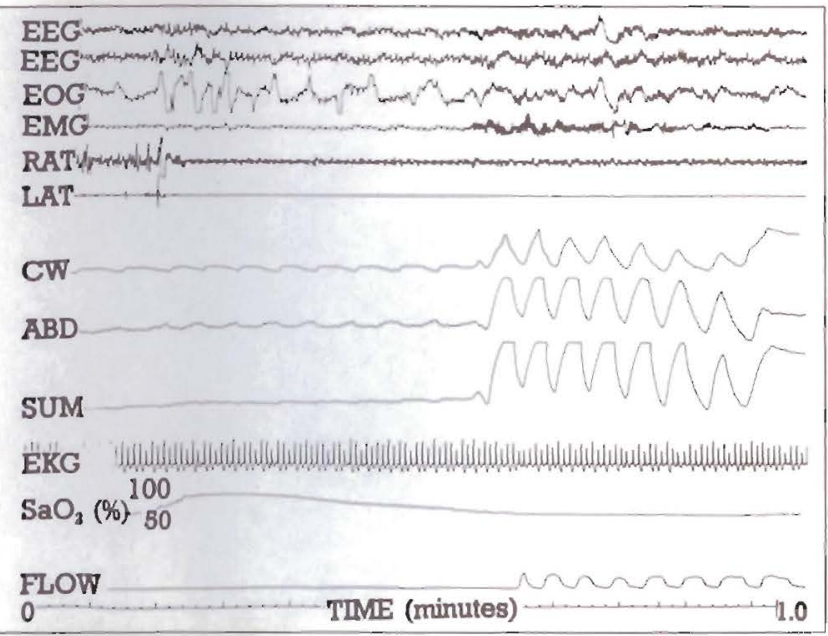

Figure 2) One minute of rapid eye movement sleep demonstrating obstructive apnea. EEG Electroencephalogram; EOG Electro-oculogram; EMG Submental electromyogram; RAT and LAT Right and left anterior tibialis EMG; CW Chest wall movement; $A B D$ Abdominal wall movement; SUM Sum of CW and ABD; EKG Electrocardiogram; $\mathrm{SaO}_{2}$ Oxygen saturation; FLOW Airflow at nose and mouth

functional residual capacity $2.97 \mathrm{~L}$ ( $77 \%$ predicted), and residual volume $2.30 \mathrm{~L}$ ( $93 \%$ predicted); expiratory reserve volume was $48 \%$ of predicted, presumably due to the patient's morbid obesity. Single-breath carbon monoxide uptake was $20.9 \mathrm{~mL} / \mathrm{min} / \mathrm{mmHg}$ (78\% predicted), and $98 \%$ predicted when corrected for single-breath lung volume. Arterial oxygen saturation by ear oximetry was $95 \%$ at rest with no change during stair climbing.

Cardiac function: A 12-lead ECG revealed atrial fibrillation with a ventricular rate of $150 \mathrm{beats} / \mathrm{min}$. Transthoracic echocardiography revealed a dilated left ventricle with severe global hypokinesis. Left ventricular ejection fraction was estimated between 20 and 30\% (normal greater than 60\%). This was confirmed by radionuclide angiography, which revealed a left ventricular ejection fraction of $16 \%$. In addition, echocardiography revealed that the ascending aorta was dilated, and aortic dissection was suspected.

Radiology: Chest radiography showed cardiomegaly and an anterior mediastinal mass (Figure 4). Further evaluation by computerized tomography confirmed the presence of a very large dissecting aneurysm in the ascending aorta.

Operation: Surgical repair was recommended since it was felt there was a significant risk of aortic rupture. Following a midline sternotomy, a $12 \mathrm{~cm}$ dissecting aneurysm of the ascending aorta was found that was compressing the vena cava and displacing the heart inferiorly and laterally to the left. The surgeon also reported evidence of old pericarditis. A Dacron tube graft replaced the abnormal aorta, and once the aneurysm was removed, the heart moved back to a normal position. Pathological examination of the resected aorta subsequently revealed a double-barrel lumen with fibrointimal hyperplasia consistent with chronic medial wall dissection.

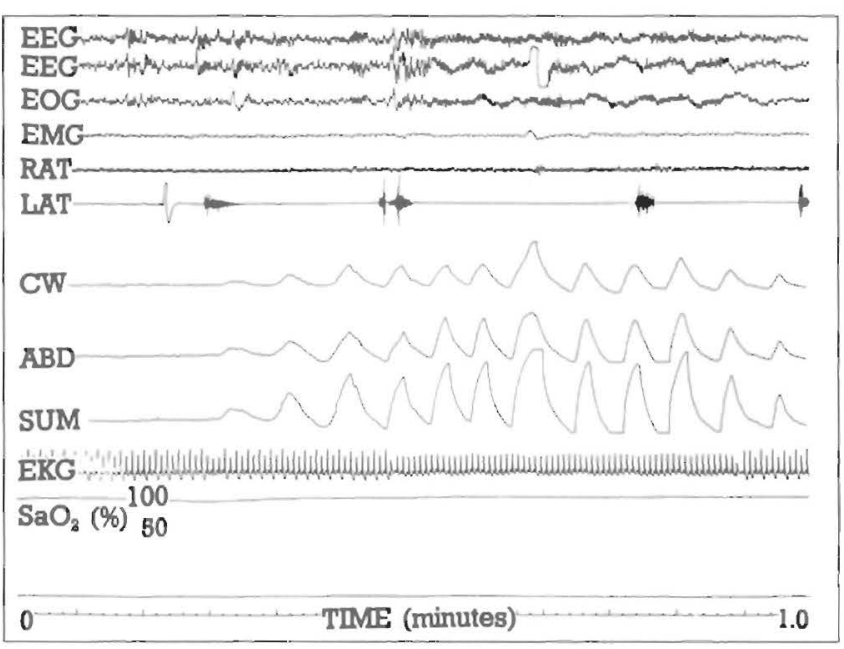

Figure 3) One minue of nonrapid eye movement sleep on nasal continuous positive airway pressure demonstrating central apnea followed by hyperpnea with a crescendo/decrescendo pattern. EEG Electroencephalogram: EOG Electro-oculogram; EMG Submental electromyogram: RAT and LAT Right and left anterior tibialis EMG: CW Chest wall movement: ABD Abdominal wall movement; SUM Sum of $C W$ and $A B D ; E K G$ Electrocardiogram: $\mathrm{SaO}_{2}$ Oxygen saturation

Progress: Postoperatively, the patient diuresed rapidly and there was a marked reduction in his facial size. In retrospect, it was apparent that he had significant superior vena caval obstruction. He reverted spontaneously to sinus rhythm. Resting radionuclide angiography two weeks following surgery revealed a left ventricular ejection fraction of $54 \%$ with persistent left ventricular enlargement. He was discharged from hospital 25 days following his surgery on enalapril 10 mg daily, enteric coated acetylsalicylic acid, and salt and calorie restriction. Congestive heart failure had resolved and he did not require diuretic therapy.

Over the next year, he continued to use $10 \mathrm{cmH}_{2} \mathrm{O}$ nasal CPAP during sleep. There was a dramatic improvement in his sleep quality and daytime function with complete resolution of hypersomnolence. Although he remained obese, his weight fell to $118 \mathrm{~kg}$ (body mass index $38.5 \mathrm{~kg} / \mathrm{m}^{2}$ ) with a reduction in his neck circumference to $47 \mathrm{~cm}$. Clinically, he remained free of heart failure although atrial fibrillation returned, requiring treatment with digoxin and amiodarone, which converted him to normal sinus rhythm. He maintained normal left ventricular systolic function although he had persistent impairment of left ventricular diastolic function, which was attributed to hypertension and previous cardiac history. Overnight polysomnography was repeated 13 months following repair of his aortic aneurysm (Table 1). The study was performed on and off nasal CPAP. Off nasal CPAP, he continued to have significant OSA with associated hypoxemia and sleep disruption. However, there was no evidence of $\mathrm{CSR}$ and $\mathrm{tCO}_{2}$ was elevated compared with the relative hypocapnia he experienced during the first sleep study. During the second part of the study, his OSA was corrected by $10 \mathrm{cmH}_{2} \mathrm{O}$ nasal CPAP. The patient remains well three years following his initial presentation. 

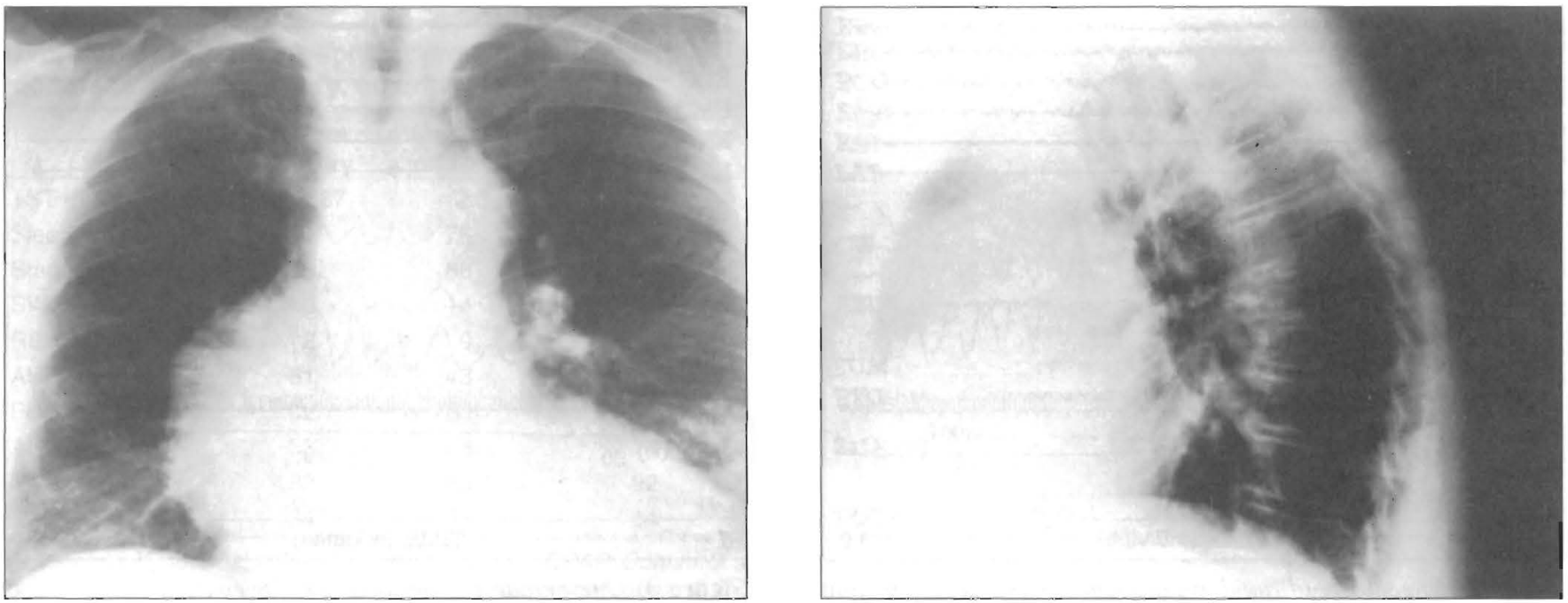

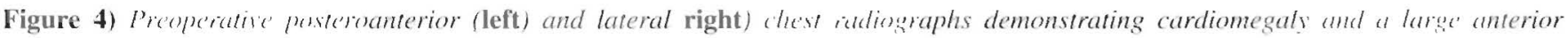
mediastinal mass

\section{DISCUSSION}

The patient described here had clinical and polysomnographic evidence of both OSA and CSR. The long history of loud snoring, witnessed apneas and excessive daytime sleepiness are consistent with OSA, and the recent history of congestive heart failure predisposed the patient to develop CSR. Although intermittent paradoxical breathing was present throughout the sleep study, obstructive apnea was most obvious during REM sleep (Figure 2), the sleep stage during which upper airway occlusion is most likely to occur. CSR was only seen during stage I and 2 NREM sleep (Figure I) and became more obvious once the upper airway was stabilized by nasal CPAP (Figure 3). The pathogenesis of CSR is closely related to destabilization of the metabolic control system, which is the primary regulator of ventilation during NREM sleep (6). Hence, the relationship between sleep stage and sleep-disordered breathing in our patient is consistent with the current understanding of the pathogenesis of these two different forms of sleep apnea.

The unique feature of this case is the potential interaction between sleep apnea and antic dissection and the role they may have played in each others' pathogenesis. It has been proposed by our cardiovascular colleagues that the patient ${ }^{\circ}$ s itortic dissection started during the episode of acute pericarditis seven years previously. In addition, he was hypertensive, which is a known risk factor for the development of aortic dissection. However, there was also clinical and polysomnographic evidence of OSA which, historically, had been present for 40 years. Obstructive apnea is associated with large negative intrathoracic pressure swings due to forced inspiration against an occluded upper airway (7). Each apnea generates a substantial pressure gradient across the left ventricle and aortic wall. Obstructive apneas are also known to cause acute elevation of systemic blood pressure during sleep (8). It is possible that this repetitive increase in the pressure gradient across the aortic wall and the intermittent rise in systemic blood pressure over many years may have extended the arortic dissection that commenced during the episode of acute pericarditis.

In addition to OSA, the patient also had polysomnographic evidence of CSR (Figures I and 3). Since the patient also had congestive heart failure, it is reasonable to assume that his CSR was caused by left ventricular dysfunction. There were many risk factors for the development of left ventricular dysfunction in this patient including hypertension, obesity, alcohol consumption and previous pericarditis (9). In addition, OSA may have exacerbated pre-existing left ventricular dysfunction (3) although it is unlikely to have caused it de novo (I0). However, we believe that the most significant caluse of impaired left ventricular function was vena caval obstruction caused by a large dissecting aneurysm of the ascending iorta. It is likely that venous return was critically reduced not only by compression of the vena cava but also by displacement of the heart. This was supported by the intraoperative lindings, clinical features of superior vena caval obstruction and the rapid clinical and echocardiographic improvement following surgery. The patient's continued improvement and the absence of CSR three years following surgery further support these suggestions.

\section{CONCLUSIONS}

We report a patient with coexisting dissecting aneurysm of the aorta and sleep apnea. We believe that vena caval obstruction associated with the aneurysm caused CSR during sleep. Furthermore, we speculate that long-standing OSA may have contributed to the development of aortic dissection.

ACKNOWLEDGEMENTS: The authors thank Llyio (iarcial lin typing the manuscript.

\section{REFERENCES}

1. Cherniak NS, Longobardo GS. Cheyne-Stokes breathing: an instability in physiologic control. N Engl J Mad 1973:288:952-7. 
2. Yamashiro Y, Kryger M. Sleep in heart failure. Sleep 1993:16:513-23.

3. Malone S, Liu P, Halloway R, Rutherford R, Xie A, Bradley TD. Obstructive sleep apnea in patients with dilated cardiomyopathy: effects of continuous positive airway pressure. Lancet 1991:338:1480-4.

4. Kales $A$. Rechtschaffen A, eds. A Manual of Standardized Terminology, Techniques and Scoring System for Sleep Stages of Human Subjects. NIH publication no. 204. Bethesda: National Institute of Neurological Disease and Blindness, 1968.

5. American Sleep Disorders Association. The International Classification of Sleep Disorders: Diagnostic and Coding Manual. Lawrence: Allen Press Inc, 1990.

6. Hanly P, Zuberi N, Gray R. Pathogenesis of Cheyne-Stokes respiration in patients with congestive heart failure: relationship to arterial $\mathrm{PCO}_{2}$. Chest 1993:104:1079-\$4.

7. Marrone O, Bellia V, Ferrara G, Milone F, Romano L. Salvaggio A, et al. Transmural pressure measurements. Importance in the assessment of pulmonary hypertension in obstructive sleep apneas. Chest 1989;95:338-42.

8. Parish JM, Sheppard JW. Cardiovascular effects of sleep disorders. Chest 1990;97:220-6.

9. Braunwald E. Clinical manifestations of heart failure. In: Braunwald E. ed. Heart Disease. Philadelphia: WB Saunders Co, 1988:471-84.

10. Hanly, P, Sasson Z, Zuberi N, Alderson M. Ventriculalr function in snorers and patients with obstructive sleep apneit. Chest 1992:102:100-5. 


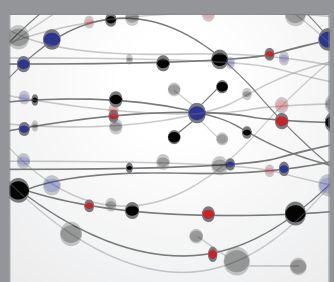

The Scientific World Journal
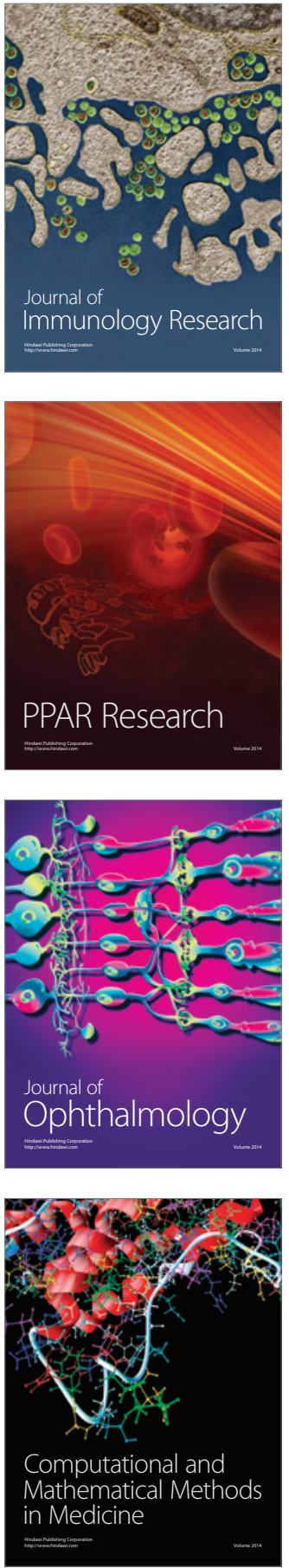

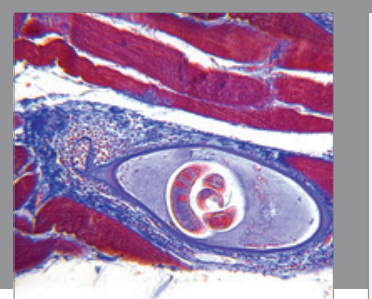

Gastroenterology Research and Practice

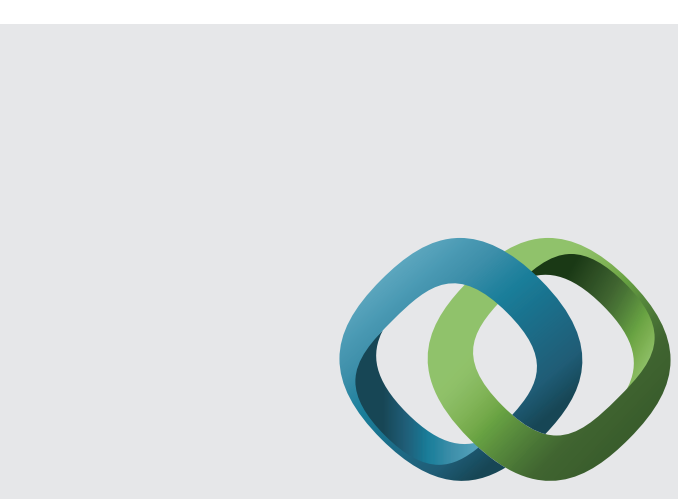

\section{Hindawi}

Submit your manuscripts at

http://www.hindawi.com
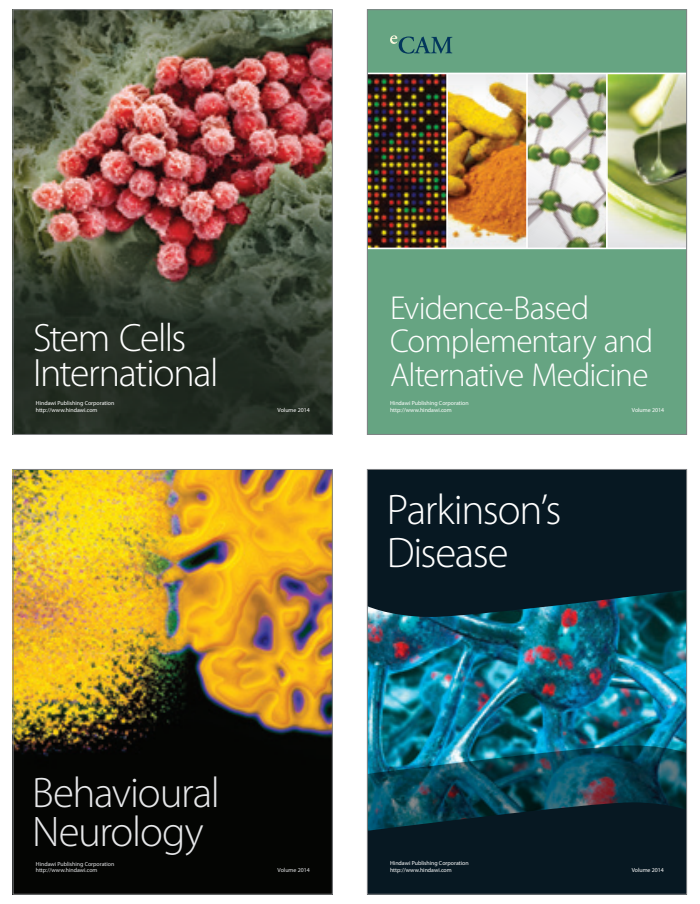
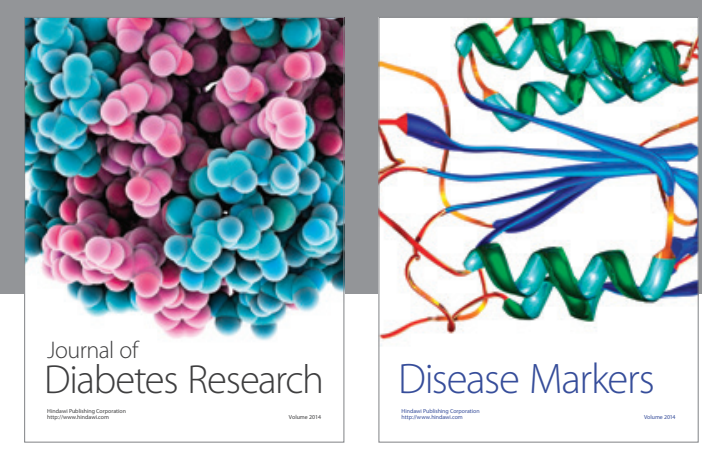

Disease Markers
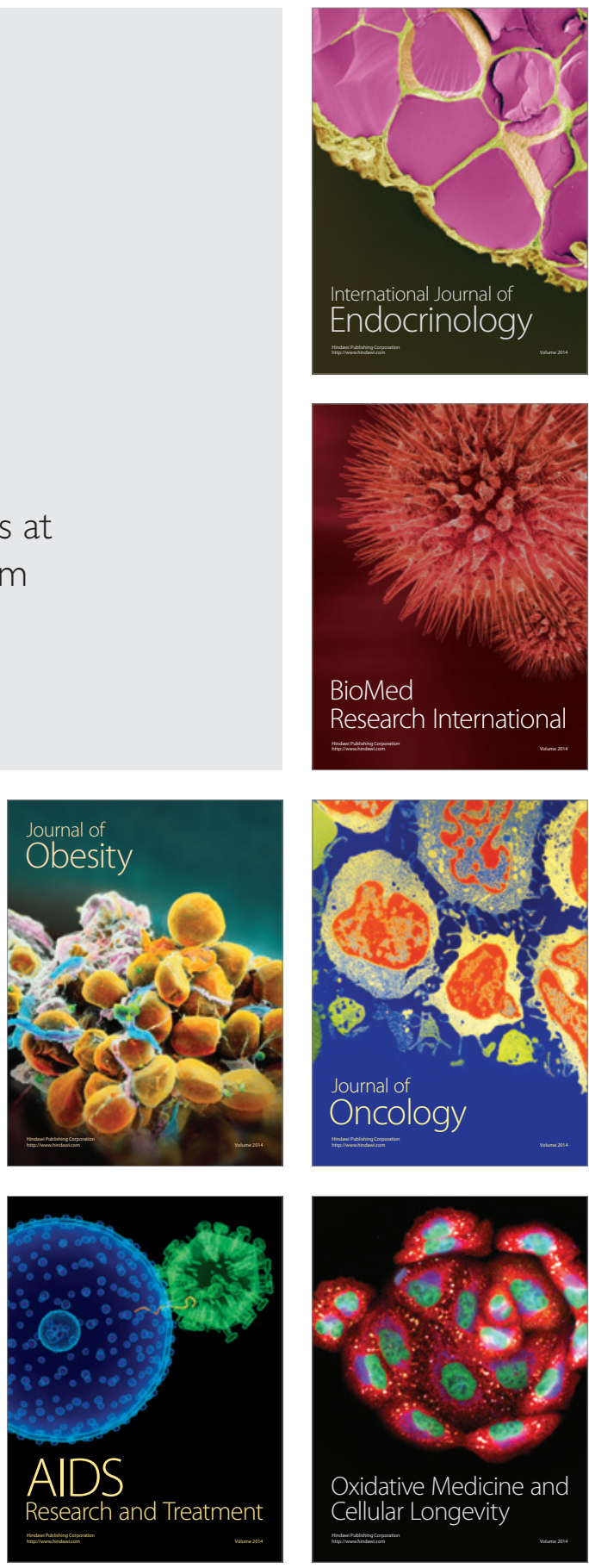